\title{
Intervenções em Terapia Cognitivo-Comportamental para Trauma Complexo: Uma Revisão Sistemática
}

\author{
Saulo Gantes Tractenberg ${ }^{1}$ \\ Luisa Zamagna Maciel \\ Bruno Kluwe Schiavon \\ Mateus Luz Levandowski \\ Christian Haag Kristensen \\ Programa de Pós-Graduação em Psicologia da Pontifícia Universidade Católica \\ do Rio Grande do Sul, Porto Alegre, RS, Brasil
}

\begin{abstract}
Resumo
Introdução: Embora a Terapia Cognitivo-Comportamental (TCC) possua evidências que demonstram sua efetividade para o tratamento de Transtornos Relacionados ao Trauma e Estresse, alguns casos específicos apresentam baixa resposta. Tais casos estão associados à exposição recorrente a eventos traumáticos, de natureza interpessoal, durante a infância. Apesar de não haver consenso sobre a nomeação destes quadros clínicos, é possível que estejam associados ao diagnóstico de Trauma Complexo (TC). Objetivo: Este estudo objetivou revisar sistematicamente evidências sobre os efeitos da TCC para TC. Método: Realizou-se buscas no MEDLINE, Embase e ISI Web of Science. Artigos com desenho de estudo clínico randomizado, estudo clínico não randomizado e estudos não controlados com intervenções em TCC para TC foram incluídos. Apenas estudos com avaliações pré e pós-intervenção foram elegíveis. Resultados: Identificou-se 369 estudos sendo 7 elegíveis para esta revisão. As intervenções abordaram vários sintomas envolvidos na proposta para diagnóstico de TC, incluindo sintomas pós-traumático, depressivos, de ansiedade e regulação emocional. Conclusão: Os artigos revisados demonstraram que intervenções terapêuticas, independente da natureza, possuem efeitos ao longo do tempo sobre sintomas de TC. A TCC revelou-se superior em comparação ao tratamento controle, sendo que tanto TCC em grupo como individual revelaram-se capazes de reduzir os sintomas de TC.
\end{abstract}

Palavras-chave: Trauma Complexo, TEPT, Terapia Cognitivo-Comportamental, DESNOS.

\section{Cognitive Behavior Therapy Interventions for Complex Trauma: A Systematic Review}

\begin{abstract}
Introduction: Although Cognitive Behavior Therapy (CBT) has been shown effectiveness for treating posttraumatic stress disorders, there are some difficults regarding specfic cases. This cases are usually associated with a history of recurrent and prolonged exposure to traumatic events during the childhood. Since there is no consensus concerning the appointment of these clinical conditions, it is suggested that such cases could represent the diagnosis of Complex Trauma (CT). Objective: This study aimed to investigate, through a systematic review, the efficacy of CBT for CT. Method: The searches were conducted in Pubmed, Embase and ISI Web of Science. Articles that described randomized controlled,
\end{abstract}

Endereço para correspondência: Pontifícia Universidade Católica do Rio Grande do Sul, Av. Ipiranga, 6681, prédio 11, sala 928, Porto Alegre, RS, Brasil 90619-900. Fone: (+55 51)3320-3633. E-mail: saulo.tractenberg@, acad.pucrs.br 
non-randomized controlled and non-controlled studies which had interventions based on CBT for CT symptoms were included.Results: The search identified 369 studies, and 7 were selected. The focus of CBT interventions comprised a variety of symptoms that have been proposed for the CT diagnosis, including posttraumatic and depressive symptoms, anxiety and emotional regulation. Conclusion: The results suggest that therapeutic interventions, regardless of type, have an effect over time on CT symptoms. CBT was more efficient compared to the control treatments. Both individual and group CBT were able to reduce CT symptoms.

Keywords: DESNOS, PTSD, Cognitive Behavioral Therapy, Complex Trauma.

\section{Las Intervenciones en Terapia Cognitivo-Conductual para Trauma Complejo: Una Revisión Sistemática}

\section{Resumen}

Introducción: A pesar de la Terapia Cognitivo-Conductual (TCC) tiener un cuerpo de evidencia que demuestra la eficacia para el tratamiento de desórdenes de estrés y trauma, algunos casos específicos tienen una baja respuesta. Estos casos están asociados con la exposición repetida a los acontecimientos traumáticos, de carácter interpersonal, durante la infancia. Aunque no existe un consenso en cuanto a la designación de estos cuadros clínicos, es posible que se asocie con un diagnóstico llamado Trauma Complejo (TC). Meta: Este estudio tuvo como objetivo investigar, a través de la revisión sistemática, estudios sobre los efectos de las intervenciones en la TCC para el TC. Método: Se realizaron búsquedas en MEDLINE, EMBASE, ISI Web of Science. Se incluyeron los artículos con diseño aleatorio estudio clínico, estudio clínico no aleatorizado y estudios no controlados con intervenciones en la TCC para la CT. Sólo los estudios con pre y post-intervención fueron elegibles. Resultados: Se identificaron 369 estudios y son 7 electos para esta revisión. Las intervenciones discutieron diversos síntomas de TC, incluyendo los síntomas postraumáticos, depresivo, de ansiedad y regulación emocional. Conclusión: Artículos revisados han demostrado que las intervenciones terapéuticas, independientemente de la naturaleza, tienen un efecto en el tiempo de los síntomas de la CT. La TCC ha demostrado ser superior en comparación con el tratamiento control. Tanto la TCC individual y grupal demostró ser capaz de reducir los síntomas de TC.

Palabras-clave: Trauma Complejo, Trastorno de Estrés Postraumático, Terapia Cognitivo-Conductual, DESNOS.

A exposição recorrente e prolongada a múltiplos eventos traumáticos de natureza interpessoal, como por exemplo, situações de abuso e violência familiar, negligência de cuidados físicos e emocionais durante a infância, pode levar os indivíduos a apresentarem um quadro clínico complexo (Herman, 2012; Pelcovitz et al., 1997; van der Kolk, Roth, Pelcovitz, Sunday, \& Spinazzola, 2005). Tal quadro difere-se dos sintomas descritos pela edição anterior do Manual Diagnóstico dos Transtornos Mentais (DSM) para o Transtorno de Estresse Pós-Traumático (TEPT; American Psychiatric Association [APA], 1994) e também dos sintomas estabelecidos pela $5^{\text {a }}$ edição (DSM-V) para os Transtornos Relaciona- dos ao Trauma e Estresse (APA, 2013). Dentre o conjunto de sintomas relatados por estes indivíduos pode-se destacar a presença de sintomatologia depressiva intensa, dificuldades de modular sentimentos de raiva, recorrentes episódios dissociativos e de perturbação da identidade, incapacidade de confiar nos outros, comportamentos autodestrutivos, descontrole de impulsos e instabilidade nos relacionamentos interpessoais, além de dores crônicas e alterações no funcionamento cognitivo (Cloitre et al., 2011; McLean \& Gallop, 2003; van der Kolk et al., 2005).

Em razão de tais sintomas irem além da caracterização do TEPT, diversos autores defendem a necessidade de inclusão de uma nova definição 
nos manuais diagnósticos que contemple esses sintomas distintos do diagnóstico dos transtornos pós-traumáticos (Bryant, 2012; Herman, 2012; Pelcovitz et al., 1997; Roth, Newman, Pelcovitz, van der Kolk, \& Mandel, 1997). Tal proposta fundamenta-se em observações clínicas que apontam para diferenças marcadas nas reações pós-traumáticas de indivíduos que sofreram um trauma único (e.g., situações de assalto, estupros e acidentes automobilísticos) em comparação a indivíduos que sofreram múltiplos traumas de natureza interpessoal ao longo do seu desenvolvimento, principalmente por parte dos cuidadores os quais espera-se que sejam fontes de segurança, proteção e estabilidade (Courtois, Ford, \& Cloitre, 2009; Viola, Kluwe-Schiavon, Renner, \& Grassi-Oliveira, 2011). Uma recente meta-análise (Teicher \& Samson, 2013), demonstrou que eventos estressores na infância podem influenciar a expressão fenotípica de diferentes psicopatologias (e.g., Transtornos de Humor, TEPT, Transtornos de Ansiedade e Transtornos por uso de Substâncias), sendo um possível responsável por distinções clínicas e neurobiológicas que tornam indivíduos com história de estresse precoce um subtipo específico dentro de um mesmo desfecho psiquiátrico. Sabe-se também que uma maior recorrência de traumas ao longo do desenvolvimento representa um agravamento na condição clínica dos pacientes que desenvolvem transtornos pós-traumáticos na vida adulta (Cloitre et al., 2009).

Herman (1992) foi um dos pioneiros na proposta de uma categorização para tais manifestações complexas, denominando-as de Transtorno de Estresse Extremo sem outra especificação (DESNOS, em inglês). Para o pesquisador o diagnóstico de DESNOS seria caracterizado por um agrupamento de 27 sintomas em 7 categorias relacionadas à (a) alterações na regulação emocional e no controle de impulsos; (b) alterações na atenção e na consciência; (c) alterações na auto-percepção; (d) alterações perceptivas relacionadas ao agressor/abusador; (e) alterações nas relações interpessoais; (f) somatização e (g) alterações nos sistemas de significados. Através de sua categorização, os quadros pós-traumáticos não mais seriam avaliados sob a perspectiva de uma forma única de manifestação pós-traumáti- ca, passando a serem considerados a partir da natureza do evento traumático, ou seja seria necessário uma distinção entre TEPT simples (evento único) e TEPT complexo (múltiplos eventos durante períodos sensíveis do desenvolvimento). A caracterização conceitual de DESNOS fez com que alguns estudos começassem a ser publicados considerando tal diagnóstico (Cloitre, Stovall-McClough, Miranda, \& Chemtob, 2004; Roth et al., 1997; van der Kolk et al., 1996; van der Kolk et al., 2005), incluindo uma proposta de entrevista estruturada para a avaliação dos sintomas de DESNOS, a Structured Interview for Disorders of Extreme Stress (SIDES; Pelcovitz et al., 1997).

Embora exista uma proposta teórica para a caracterização de DESNOS como um transtorno distinto do diagnóstico atual de TEPT, ainda existem divergências teóricas e conceituais. Para aqueles que são desfavoráveis a proposição de um diagnóstico complexo, o DESNOS representaria uma sobreposição entre sintomas pertencentes a diferentes trasntornos de eixo I e II, como TEPT, Transtorno Depressivo Maior (TDM) e Transtorno de Personalidade Borderline (TPB) (Resick et al., 2012). Por outro lado, os que defendem a proposta diagnóstica argumentam que o mesmo representa uma extensão ao diagnóstico de TEPT e poderia auxiliar os clínicos na elaboração de estratégias de intervenção psicoterápicas e farmacológicas efetivas, uma vez que o foco do tratamento seria sobre os sintomas de TC e não apenas sobre os sintomas pós-traumáticos subjacentes ao TEPT (revivência, evitação e alterações no humor e hiperexcitabilidade; Bryant, 2012; Cloitre et al., 2011; Cloitre et al., 2010; Herman, 2012; van der Kolk et al., 2005). A idéia desses autores sustenta-se nas dificuldades no tratamento destes pacientes através de protocolos de intervenções consolidados para o tratamento indivíduos com TEPT (Courtois et al., 2009; Teicher \& Samson, 2013), como as terapias de exposição (Butler, Chapman, Forman, \& Beck, 2006).

Não somente a proposta diagnóstica de DESNOS, como também a sua nomenclatura permanece em debate. A antiga proposta de Herman (1992) para um diagnóstico complexo é similar ao que alguns autores denominam de TC, 
Transtorno de Estresse Pós-traumático do Desenvolvimento ou ainda Transtorno do Trauma Desenvolvimental (van der Kolk, 2005). Apesar das diferenças em sua denominação, prevalece a caracterização de um quadro clínico com sintomas pós-traumáticos, afetivos e interpessoais decorrente da presença de traumas repetidos durante períodos precoces do desenvolvimento (Cloitre et al., 2011; Herman, 2012; McLean \& Gallop, 2003). Assim, entende-se que independente dos debates teóricos e conceituais em relação ao diagnóstico DESNOS ou TC, a importância de considerar tais sintomas como um quadro clínico distinto advêm da prática clínica que demonstra a refrateriedade de tais casos quando abordados através de protocolos baseados em abordagens cognitivas e comportamentais (e.g., Terapia Cognitivo-Comportamental, Terapia de Exposição, Terapia de Dessensibilização e Reprocessamento por Movimentos Oculares). Inclusive, têm sido sugerido que intervenções focadas no trauma, poderiam ser prejudiciais para o tratamento TC (Herman, 2012). Tendo em vista tais dificuldades, o presente artigo objetiva realizar uma revisão sistemática da literatura sobre as intervenções cognitivas e comportamentais direcionadas para o tratamento de indivíduos com diagnóstico de TC ou DESNOS.

\section{Método}

A busca por artigos foi realizada em três bases de dados: MEDLINE, Embase e ISI Web of Knowledge e segue as recomendações do checklist da Cochrane para o desenvolvimento de estratégias de busca para artigos de revisão sistemática. Foram utilizados os seguintes descritores em inglês: [CPTSD OR "Chronic Posttraumatic Stress Disorder" OR "Chronic PTSD" OR "Complex PTSD" OR "Complex Trauma" $O R$ "Disorders of Extreme Stress" OR DESNOS] AND ["Cognitive Therapy" OR "Behavior Therapy" OR "Cognitive Behavioral Therapy" OR "Group Psychotherapy" OR "Dialectical Behavior Therapy" OR "Schema Therapy"]. Os limites estabelecidos foram "somente artigos em inglês" e "somente artigo com humanos", não tendo sido aplicado quaisquer restrições relacionadas a data de publicação. As buscas foram re- alizadas no período que compreende os meses de fevereiro e março de 2015 . O critério de busca estabelecido foi a presença dos termos descritores no título, resumo ou no assunto do artigo. Foram incluídos artigos que possuíam delineamento de estudo clínico randomizado, estudo clínicos não randomizados e estudos não controlados. Os critérios de exclusão dos artigo adotados foram: (a) artigos não-empíricos; (b) estudos de caso; (c) artigos que não possuíam intervenção em terapias cognitivo-comportamentais (TCC); (d) artigos que não possuíam indivíduos com diagnóstico de TC ou DESNOS para avaliação da intervenção; (e) artigos com intervenção mas que não possuiam medidas pré e pós tratamentoe (f) artigos com amostra repetida.

O procedimento de seleção dos estudos ocorreu por intermédio de dois avaliadores independentes (SGT e LZM) que deram seus pareceres sobre a possibilidade de inclusão ou exclusão dos artigos na revisão. As divergências entre os dois avaliadores foram analisadas e discutidas por um terceiro avaliador com vasta experiência no tema de trauma e estresse (CHK). Apenas dois artigos necessitaram de revisão pelo terceiro avaliador, tendo sido ambos excluídos pois se tratavam de estudos com amostras diagnosticadas apenas com TEPT crônico. Nos casos em que a exclusão ou inclusão não foi possível através da avaliação do título ou do resumo, realizou-se a avaliação texto completo $(n=1)$. Além disso, foi realizada busca na lista de referência de cada artigo incluído na revisão para possível inclusão de artigos que não foram contemplados através da busca nas bases de dados $(n=1)$. A qualidade dos artigos que possuíam o desenho de estudo clínico randomizado foi mensurada através da escala JADAD de cinco pontos. Foram considerados pontos para os seguintes critérios (a) Randomização dos participantes; (b) Randomização realizada adequadamente; (c) Avaliadores eram cegos para o tipo de tratamento do paciente; (d) $\mathrm{O}$ método em que o critério 3 foi aplicado era adequado; (e) Descrição adequada dos pacientes que abandonaram o estudo e/ou tratamento. Nos casos em que era recomendado pela escala a retirada de pontos caso o estudo não atendesse determinado critério, optou-se por não realizar tal desconto. Ainda, em um caso houve a necessida- 
de de realizar a avaliação dos critérios JADAD utilizando-se como fonte de informação um estudo prévio dos autores, uma vez que a amostra com TC era derivada de um estudo maior com amostra de indivíduos com inicialmente diagnosticados com TEPT.

\section{Resultados}

As buscas realizadas identificaram um total de 369 artigos dos quais 7 artigos foram selecionados para a revisão (Figura 1).

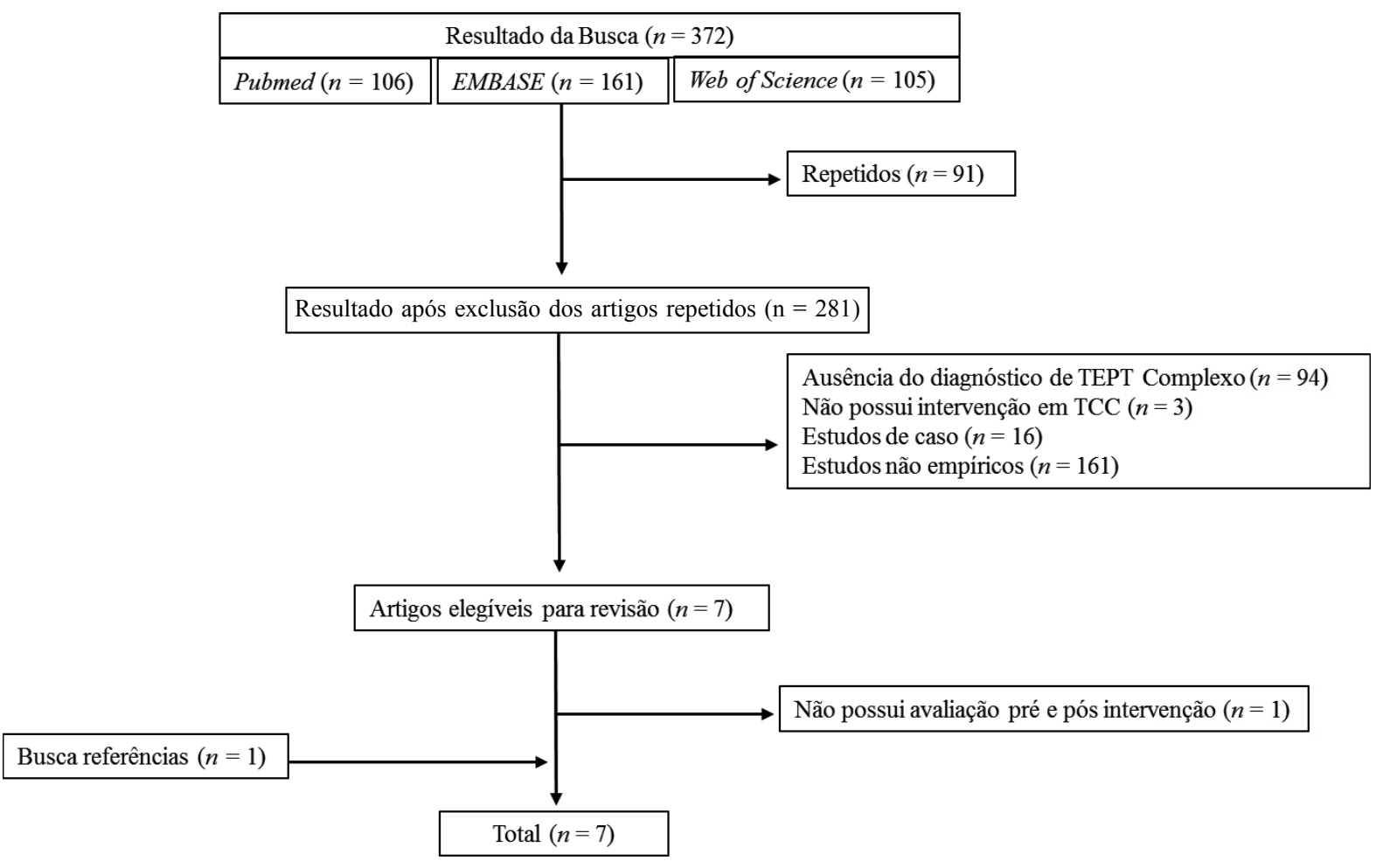

Figura 1. Fluxograma da revisão sistemática.

A qualidade metológica dos estudos selecionados para revisão são apresentadas na tabela 1 em conjunto com as características dos estudos. Entre os artigos selecionados para revisão, 3 eram estudos clínicos randomizados (Cohen \& Hien, 2006; Dorrepaal et al., 2012; Resick, Nishith, \& Griffin, 2003; Zlotnick et al., 1997), 2 eram estudos clínicos não randomizado (Trappler \& Newville, 2007) e 2 eram estudos não controlados (D'Andrea \& Pole, 2012; Dorrepaal et al., 2010). A escala JADAD foi utilizada para avaliação dos quatro estudos que possuíam desenhos de estudos clínicos randomizados. No que se refere as características dos estudos, foram reportadas informações sobre o sexo, idade e dignóstico psiquiátrico de todos participantes do estudo. Além disso, foram descritos os sintomas avaliados, bem como os intrumentos utilizados para a avaliação. Somente dois estudos (Dorre- paal et al., 2010; Dorrepaal et al., 2012) utilizaram um instrumento próprio para avaliação de sintomas de TC, a SIDES. Os demais estudos consideraram como parâmetro de avaliação dos sintomas de TC o resultado proveniente de diferentes escalas de sintomas psiquiátricos, como escalas de sintomas pós-traumáticos, de depressão, ansiedade, impulsividade, dissociação, funcionamento sexual, queixas somáticas, funcionamento cognitivo e padrão de uso de substância. Especificamente, para avaliação dos sintomas pós-traumáticos houve uma variabilidade nos instrumentos selecionados pelos estudos, sendo a Davidson Trauma Scale (DTS) e a Clinical Administered PTSD Scale (CAPS) os instrumentos mais frequentemente utilizados $(n=3)$. As outras escalas utilizadas foram a Trauma Symtom Inventory (TSI), Impact of Event Scale (IES) e a Posttraumatic Stress Disorder Checklist (PCL). 


\section{Tabela 1}

Qualidade metodológica e características dos artigos

\begin{tabular}{|c|c|c|c|}
\hline ID JADAD & Autor (ano) & $\begin{array}{c}\text { Total da amostra } \\
{[\text { media de idade }(D P)]}\end{array}$ & Mensurações \\
\hline
\end{tabular}

Estudos controlados randomizados

2 Dorrepaal et al.

71 mulheres

com TEPT/TC

$[38,7(10,5)]$

$02 *$

3

Resick et al.

(2003)

121 mulheres

com TEPT

$[33,1(9,85)]$

03

2

Zlotnick et al. (1997)

48 muheres com TEPT/TC

[39 (9,59)]

Estudos controlados não randomizados

$\begin{array}{cc}\text { 04* } 1 \quad \begin{array}{cc}\text { Cohen \& Hien } \\ \text { (2006) }\end{array} & 107 \text { mulheres } \\ & \text { com TEPT e TUS } \\ & {[37,92(9,82)]}\end{array}$

05

Trappler

\& Newville

(2007)

Estudos não controlados

06

Dorrepaal et al.

(2010)

36 mulheres

com TEPT/TC

$[34,1(8,3)]$

$07 \quad$ - $\quad \begin{array}{cc}\text { D'Andrea } & 27 \text { mulheres } \\ \text { \& Pole }(2012) & \text { com TEPT }\end{array}$

$[38,1(13,4)]$

\author{
Sintomas de TEPT: DTS \\ Diagnóstico de TEPT complexo: SIDES$^{1}$ \\ Sintomas de TC: SIDES ${ }^{2}$ \\ Sintomas de TPB: BPDSI-IV \\ Sintomas Dissociativoss: DES \\ Diagnóstico de TEPT: CAPS \\ Sintomas depressivos: BDI \\ Exposição ao trauma: STI \\ Sintomas do trauma: TSI \\ Diagnóstico de TEPT: CAPS \\ Sintomas de TEPT: DTS e sub-escala CR-PTSD da SCL-90 \\ Sintomas dissociativos: DES \\ Trauma na infância: CTQ \\ Diferença entre intervenções: PTF
}

Diagnóstico de TEPT: CAPS

Exposição ao trauma: LEC

Diagnóstico de TUS: SCID-I

Padrões de uso de substância e funcionamento social: ASI

Sintomas de depressão: HDRS

Sintomas dissociativos: DES

Funcionamento sexual: sub-escala DBS da TSI

Reações ao estresse: IES

Depressão, Ansiedade, Alucinações e outros sintomas psiquiátricos: BPRS
Gravidade dos sintomas de TEPT: DTS

Sintomas de TPB: BPDSI-IV

Sintomas dissociativos: DES

Sintomas gerais de psicopatologia: SCL-90

Sintomas depressivos: BDI

Diagnóstico de TC: SIDES ${ }^{1}$

Diagnóstico de TEPT: SCID-I

Sintomas depressivos e de ansiedade e sensibilidade interpessoal: BSI

Experiência dissociativa: DES

Sintomas de TEPT: PCL

Exposição ao Trauma: THQ

Atividade no Sistema Nervoso Parassimpático: RSA

Nota. ASI = Addiction Severity Index; BDI = Beck Depression Inventory; BPDSI-IV = Borderline Personality Disorder Severity Index-IV; BPRS = Brief Psychiatric Rating Scale; BSI = Brief Symptom Inventory; CAPS = Clinical Administered PTSD Scale; CTQ = Childhood Trauma Questionnaire; DBS = Dysfunctional Sexual Behavior Scale; DES = Dissociative Experiences Scale; DTS = Davidson Trauma Scale; HDRS = Hamilton Depression Rating Scale; IES = Impact of Event Scale; LEC = Life Event Checklist $; \mathrm{PCL}=$ Posttraumatic Stress Disorder Checklist $; \mathrm{RSA}=$ Respiratory Sinus Arrhythmia; $\mathrm{SC}=$ Skin Conductance; SCID-I = Structured Clinical Interview for DSM Disorders; SCL-90 = Symptom Checklist-90-Revised; SIDES $^{1}$ $=$ Structured Interview for Disorders of Extreme Stress - original 48-item version; SIDES $^{2}=$ Structured Interview for Disorders of Extreme Stress - revised 37-item version; STI = Standardized Trauma Interview; TSI = Trauma Symtom Inventory; THQ = Trauma History Questionnaire; $\mathrm{nr}=$ não reportado.

*A amostra destes estudos deriva de um ensaio clínico anterior que não possuia enfoque no tratamento de sintomas relacionados ao diagnóstico de Trauma Complexo. Se o estudo elegível para a revisão não reportou informações necessárias para o escore de JADAD, o escore foi baseado nos críterios de inclusão do ensaio clínico original. 
As características das intervenções, como tempo de duração semanal da sessão, tempo to- tal de duração da intervenção, modalidade (individual ou em grupo) e foco da intervenção estão descritos na Tabela 2.

Tabela 2

Características da intervenção

\begin{tabular}{|c|c|c|c|c|c|c|}
\hline ID & Int. & $\begin{array}{c}\text { Sessões/semana } \\
\text { (horas/semana) }\end{array}$ & $\begin{array}{l}\text { Total } \\
\text { horas }\end{array}$ & $\begin{array}{l}\text { Tipo } \\
\text { sessão }\end{array}$ & $\begin{array}{l}\text { Número de } \\
\text { participantes }\end{array}$ & Foco da intervenção \\
\hline 01 & $\begin{array}{l}\text { EXP } \\
\text { TAU }\end{array}$ & $1(2)$ & 40 & G & $8-12$ & $\begin{array}{c}\text { (1) Sintomas nucleares de TC } \\
\text { (2) Senso de domínio cognitivo } \\
\text { (3) Identificar e modificar comportamentos } \\
\text { disfuncionais, pensamentos e crenças sobre o trauma } \\
\text { (4) Aquisição de auto-regulação, crenças adaptativas } \\
\text { e assertividade } \\
\text { (5) Restaurar a esperança e reduzir a vergonha, culpa } \\
\text { e isolamento }\end{array}$ \\
\hline 02 & $\begin{array}{l}\text { TCP } \\
\text { EP } \\
\text { LE }\end{array}$ & $\begin{array}{c}2(1.08) \\
2(1.44) \\
-\end{array}$ & $\begin{array}{c}13 \\
13 \\
-\end{array}$ & I & - & $\begin{array}{l}\text { (1) Sintomas de TEPT } \\
\text { (2) Sintomas depressivos } \\
\text { (3) Dissociação } \\
\text { (4) Auto-referência } \\
\text { (5) Comportamentos de tensão }\end{array}$ \\
\hline 03 & $\begin{array}{l}\text { MA } \\
\text { LE }\end{array}$ & $\begin{array}{l}1(2) \\
-\end{array}$ & $\begin{array}{c}30 \\
-\end{array}$ & $\mathrm{G}$ & $6-8$ & $\begin{array}{l}\text { (1) Sintomas de TEPT } \\
\text { (2) Dissociação } \\
\text { (3) Identificação das emoções } \\
\text { (3) Manejo da raiva } \\
\text { (5) Manejo da ansiedade }\end{array}$ \\
\hline 04 & $\begin{array}{c}\text { TCC } \\
\text { LE/TAU }\end{array}$ & $\begin{array}{l}2(1) \\
2(1)\end{array}$ & 24 & I & - & $\begin{array}{l}\text { (1) Sintomas de TEPT } \\
\text { (2) Sintomas de abstinência } \\
\text { (3) Depressão } \\
\text { (4) Dissociação } \\
\text { (5) Funcionamento social } \\
\text { (6) Funcionamento sexual }\end{array}$ \\
\hline 05 & $\begin{array}{c}\text { TCCG } \\
\text { PA }\end{array}$ & $1(1.5)$ & 18 & G & $\mathrm{nr}$ & $\begin{array}{l}\text { (1) Estabelecimento de confiança } \\
\text { (2) Identificação de sentimentos, gatilhos, pensamentos } \\
\text { e estratégias de regulação emocional } \\
\text { (3) Desenvolvimento de habilidades de regulação } \\
\text { emocional } \\
\text { (4) Estratégias comportamentais }\end{array}$ \\
\hline 06 & TGE/TAU & $1(2)$ & 40 & G & $8-12$ & $\begin{array}{l}\text { (1) Diminuir os sintomas centrais de TC } \\
\text { (2) Senso de domínio cognitivo } \\
\text { (3) Aquisição de auto-regulação, crenças adaptativas, } \\
\text { estabilidade } \\
\text { (4) Restaurar a esperança, reduzir a vergonha, culpa } \\
\text { e isolamento }\end{array}$ \\
\hline 07 & $\begin{array}{l}\text { EP } \\
\text { IS } \\
\text { PD }\end{array}$ & $\mathrm{nr}$ & $\mathrm{nr}$ & I & - & $\begin{array}{l}\text { (1) Sintomas subjetivos de TEPT: dissociação, } \\
\text { sensibilidade interpessoal, depressão e ansiedade } \\
\text { generalizada } \\
\text { (2) Viés atencional } \\
\text { (3) Memória implícita } \\
\text { (4) Psicofisiologia }\end{array}$ \\
\hline
\end{tabular}

Nota Int. $=$ Intervenções; EP = Exposição Prolongada; EXP = TGE + TAU; IS = Inoculação de Estresse; LE = Lista de Espera; $\mathrm{MR}=$ Manejo do Afeto PA = Psicoterapia de Apoio; $\mathrm{PD}=$ Terapia Psicodinâmica TAU $=$ Tratamento Usual; $\mathrm{TCC}=$ Terapia Cognitivo-Comportamental; TCCG = Terapia Cognitivo-Comportamental em Grupo; TCP = Terapia Cognitiva Processual; $\mathrm{TGE}=$ Tratamento de Grupo para Estabilização; $\mathrm{I}=$ individual e $\mathrm{G}=$ grupo; $\mathrm{nr}$ = não reportado. 
O formato das intervenções foi predominantemente grupal $(n=4)$, enquanto o formato de psicoterapia individual foi adotado por 3 estudos. Com excessão de um estudo (D'Andrea \& Pole, 2012) que não reportou dados sobre o tempo de intervenção, as intervenções variaram entre a frequência semanal de 1 a 2 vezes com encontros de duração média de 98,33 $\pm 25,62$ minutos. Com relação ao tempo total de duração do protocolo de intervenção, a média foi de 27,5 $\pm 11,23$ horas

Os estudos de Dorrepaal (Dorrepaal et al., 2010; Dorrepaal et al., 2012), Trappler e Newville (2007) e Zlotnick et al. (1997) adotaram distintos protocolos de TCC para grupos. Dorrepall seguiu uma adaptação do protocolo proposto por Zlotnick et al. (1997), que possui o objetivo de estabilizar a sintomatologia associada ao trauma, aumentando a capacidade do indivíduo em tolerar emoções intensas. Já o estudo de Trappler e Newville (2007), baseou sua intervenção no protocolo STAIR (Skill Training in Affect Regulation) desenvolvido por Cloitre, Cohen e Koenen (2006). Embora as intervenções sejam distintas no que se refere a sua estrutura e tempo de duração, ambas estão direcionadas principalmente para o aumento da capacidade de regulação emocional dos indivíduos diagnosticados com TC, além da identificação e modificação de cognições e comportamentos disfuncionais. Ainda, estes estudos, com excessão de Zlotnick et al. (1997), tiveram grupos controle ativos baseados em tratamentos usuais e terapia de suporte como parâmetros de comparação. As intervenções individuais adotadas pelos estudos de Cohen e Hien (2006), D'Andrea e Pole (2012) e Resick et al. (2003) e objetivaram a redução dos sintomas de TC, como sintomas depressivos, dissociativos, de ansiedade, disfuncionalidade sexual e alterações nas funções cognitivas relacionadas a atenção e memória. Em razão das comorbidades entre dependência de substâncias e TEPT com manifestação clínica de TC entre os participantes do estudo de Cohen e Hien (2006), a intervenção baseou-se no tratamento simultâneo de ambos os transtornos e na prevenção de recaídas. Além disso, procurou identificar os gatilhos e estratégias de coping para manejo da físsura e das recaídas. D'Andrea e Pole (2012) e Resick et al. (2003), por sua vez, adotaram protocolos de TCC consolidados para tratamento de indivíduos que experienciaram situações traumáticas, incluindo intervenções baseadas na exposição prolongada, terapia cognitiva processual e inoculação de estresse.

A Tabela 3 apresenta os principais desfechos encontrados pelos estudos elegíveis para esta revisão. Foram encontradas taxas de comorbidades que variaram entre $32,7 \%$ e $70 \%$ com diferentes transtornos psiquiátricos, incluindo TDM, TPB, Fobia Social, Transtorno por uso de Substância e Transtorno Dissociativo de Identidade nas amostras dos artigos selecionados, sendo que em Cohen e Hien (2006) e Trappler e Newville (2007) os participantes incluídos nos estudos já possuiam diagnósticos prévios de TEPT e transtorno relacionado ao uso de substância e diagnósticos de TEPT e esquizofrênia ou transtorno de personalidade esquizoafetivo, respectivamente. As principais comorbidades reportadas pelos estudos foram: (a) Transtorno de Humor Depressivo $(n=3)$, apresentando uma frequência variável entre $35 \%$ e $70 \%$; (b) Transtorno de Personalidade Borderline $(n=2)$, com taxas entre $40 \%$ e $55 \%$. Outros diagnósticos psiquiátricos também foram identificados entre as amostras, como transtornos de ansiedade e transtornos dissociativos. Além disso, observou-se uma alta frequência de situações de abuso (físico e sexual) na infância por todos estudos os quais tal aspecto foi mensurado. Os trabalhos de D'Andrea e Pole (2012), Dorrepaal et al. (2010; 2012) e Zlotnick et al. (1997) reportaram ocorrência de abuso infantil em toda a amostra. Ainda, a frequência de situações de abuso físico e/ou sexual na vida adulta demonstrou-se elevada, variando entre taxas de $35 \%$ a $96 \%$.

No que se refere aos efeitos ao longo do tempo decorrentes das intervenções em TCC, os dados convergem para uma melhora dos sintomas que compreendem o diagnóstico de TC com o passar do tempo, indicando que houve uma redução na sintomatologia entre as avaliações prée pós-intervenção (Dorrepaal et al., 2010; 2012; Resick et al., 2003; Trappler \& Newville, 2007). Dentre os sintomas que mais se diferenciaram 
entre as avaliações pré- e pós-intervenção destacam-se os sintomas de estresse pós-traumático, ansiedade, depressão e dissociação. Em Trappler e Newville (2007), no qual foi utilizado uma escala de sintomas psiquiátricos gerais também foi obtido efeito entre pré- e pós- intervenção para o escore geral no grupo de TCC. Além disso, foram observados declínio significativo nos escores das subescalas do instrumento. As subescalas relacionadas a abstinência emocional, tensão, humor depressivo, conteúdo não usual do pensamento, embotamento afetivo, excitabilidade, queixa somática, ansiedade, hostilidade, falta de cooperação e desconfiança apresentaram efeitos positivos após intervenção em TCC. Embora o artigo de D'Andrea e Pole (2012) não tenha apresentado efeito ao longo do tempo sobre os sintomas clínicos do TC, observou-se um efeito para os sintomas cognitivos (memória implícita relacionada ao trauma e viés atencional em relação a estímulos de ansiedade e relacionados ao trauma) decorrentes do TC. Para os estudos que realizaram uma segunda avaliação de seguimento ( $\mathrm{t} 2$ ), em um período de tempo determinado após a avaliação ao final da intervenção, não foram encontradas reduções significativas nos sintomas pós-traumáticos e de humor em comparação a avaliação de seguimento pós término da avaliação (t1) (Resick et al., 2012). Em comparação a avaliação de linha de base (t0) houveram reduções nas escalas psiquiátricas gerais e de sintomas pós-traumáticos (Dorrepaal et al., 2010).

Com relação ao efeito do grupo de intervenção (TCC x controle) sobre o quadro clínico de TC, os achados revelam-se dependentes do sintomas avaliados. Três estudos, Cohen e Hien (2006), Dorrepaal et al. (2012) e Zlotnick et al. (1997), compararam os efeitos do grupo experimental em relação ao grupo controle (tratamento usual e/ou lista de espera). O estudo de Cohen e Hien (2006) indicou uma melhora significativa nos sintomas de estresse pós-traumatico e no funcionamento subjacente ao uso de álcool no grupo que passou por intervenção com TCC, não sendo tal efeito identificado no grupo de tratamento usual e lista de espera. Entretanto, não houve diferenças entre os tratamentos no que se refere aos sintomas de humor, dissociação, funcionamento social e para a escala de disfunção sexual. Dorrepaal et al. (2012), por sua vez, encontrou redução significativa nos sintomas de TC para o grupo com intervenção em TCC em comparação ao grupo de tratamento usual. Já Zlotnick et al. (1997) encontrou uma melhora significativa nos sintomas pós-traumáticos e dissociativos no grupo de intervenção em comparação ao grupo de lista de espera. Os demais estudos não realizaram comparações entre os grupos de intervenção, porém D'Andrea e Pole (2012) realizou uma análise de predição de mudança e identificou que as intervenções a partir de inoculação de estresse (SI, stress inoculation em inglês) e psicodinâmica (PT) foram capazes de predizer mudanças nos sintomas de estresse pós-traumático e regulação emocional associado a estimulos do trauma, sendo que a PT foi capaz também de predizer mudança para sintomas de depressão.

\section{Discussão}

O presente estudo se propôs a revisar e descrever os efeitos de intervenções em TCC para o tratamento de indivíduos com TC. Até onde se tem conhecimento trata-se da primeira revisão a investigar de forma sistemática os achados de tratamentos baseados na TCC para indivíduos com diagnóstico ou sintomatologia associada à proposta diagnóstica de $\mathrm{TC}$ desenvolvida por Herman (1992) e defendidas por outros teóricos (Cloitre et al., 2011; McLean \& Gallop, 2003; Pelcovitz et al., 1997; van der Kolk et al., 2005). Apesar da carência no que se refere a quantidade de estudos publicados com objetivo de avaliar a efetividade da TCC para TC e, também, das limitações metodológicas que restringem a possibilidade de melhores análises e conclusões, nossos resultados suportam as evidências já consolidadas no que se refere a TCC como tratamento efetivo para transtornos pós-traumáticos (Butler et al., 2006). Assim, mesmo para quadros complexos nos quais os indivíduos apresentam histórico de traumas recorrentes e de início precoce, a TCC demonstra ser a abordagem mais indicada. 
Tbela 3

Características da amostra e principais resultados

\begin{tabular}{|c|c|c|c|c|c|}
\hline ID & Int. & $\begin{array}{c}\text { Comorbidade } \\
(\%)\end{array}$ & $\begin{array}{c}\text { Trauma na } \\
\text { Infância }(\%)\end{array}$ & $\begin{array}{l}\text { Trauma na } \\
\text { adultez }(\%)\end{array}$ & Principais resultados \\
\hline 01 & $\begin{array}{l}\text { EXP } \\
\text { TAU }\end{array}$ & $\begin{array}{c}\S \mathrm{TDM}=35,1 \\
\S \mathrm{FS}=43,4 \\
\S \mathrm{TP}=36,9 \\
\S \mathrm{TUS}=19,4 \\
\S \mathrm{TPB}=52,5 \\
\S \mathrm{TPE}=25\end{array}$ & $\begin{array}{c}\S 100 \\
\S \mathrm{AF}=62,4 \\
\S \mathrm{AS}=94,2\end{array}$ & $\begin{array}{c}\S 62,7 \\
\S \mathrm{AF}=42,7 \\
\S \mathrm{AS}=48,7\end{array}$ & 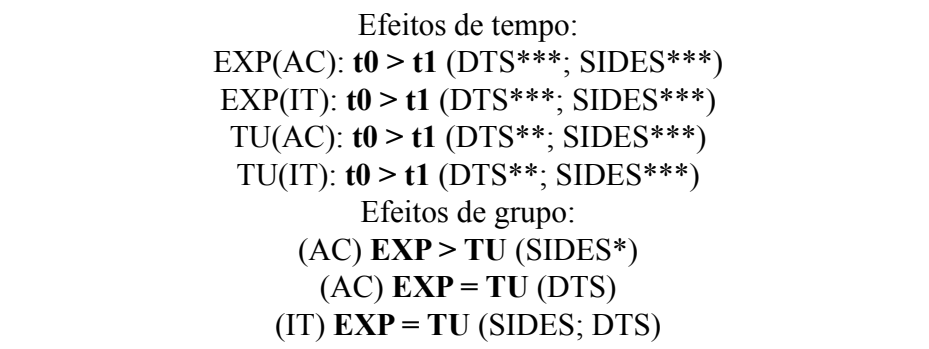 \\
\hline 02 & $\begin{array}{l}\text { TCP } \\
\text { EP } \\
\text { LE }\end{array}$ & $\mathrm{nr}$ & $\mathrm{AS}=38,8$ & $\mathrm{nr}$ & $\begin{array}{c}\text { Efeitos de tempo: } \\
\S \mathbf{t 0}>\mathbf{t} 1=\mathbf{t} 2\left(\text { CAPS } * * * ; \text { BDI } * * * ; \mathrm{TSI}^{* * *}\right) \\
\text { Efeitos de grupo: } \\
\text { TCP }=\mathbf{E P}(\mathrm{CAPS} ; \mathrm{BDI} ; \mathrm{TSI}) \\
\text { Tempo X Efeitos de Grupos: não eficaz }\end{array}$ \\
\hline 03 & $\begin{array}{l}\text { MA } \\
\text { LE }\end{array}$ & $\mathrm{nr}$ & $\S \mathrm{AS}=100$ & $\S 77$ & $\begin{array}{c}\text { Efeitos de grupo: } \\
\text { AM }>\text { LE }(\text { DTS*; CR-PTSD*; DES*) }\end{array}$ \\
\hline 04 & $\begin{array}{l}\text { TCC } \\
\text { LE/TU }\end{array}$ & $\begin{array}{c}\S \mathrm{TDM}=39,2 \\
\S \mathrm{TD}=32,7\end{array}$ & $\begin{array}{l}\S A F=60 \\
\S A S=49\end{array}$ & $\begin{array}{l}\S \mathrm{AF}=37,3 \\
\S \mathrm{AS}=35,5\end{array}$ & $\begin{array}{c}\text { Efeitos de Grupo: } \\
\text { TCC }>\text { LE/TU [CAPS**; ASI(a)*] } \\
\text { TCC }=\text { LE/TU }[\text { ASI(d); HADRS; DES; ASI(s); TSI-DSB] }\end{array}$ \\
\hline 05 & $\begin{array}{l}\text { TCCG } \\
\text { PA }\end{array}$ & $\mathrm{nr}$ & $\mathrm{nr}$ & $\mathrm{nr}$ & $\begin{array}{c}\text { Efeitos de tempo: } \\
\text { TCCG: to > t1 (BPRS***; IES**) }\end{array}$ \\
\hline 06 & $\begin{array}{l}\text { SGT/ } \\
\text { TAU }\end{array}$ & $\begin{array}{l}\S \mathrm{TDM}=70 \\
\S \mathrm{TPB}=44\end{array}$ & $\begin{array}{c}\S 100 \\
\S \mathrm{AF}=72 \\
\S \mathrm{AS}=69\end{array}$ & $\begin{array}{c}\S 75 \\
\S \mathrm{AF}=64 \\
\S \mathrm{AS}=61\end{array}$ & 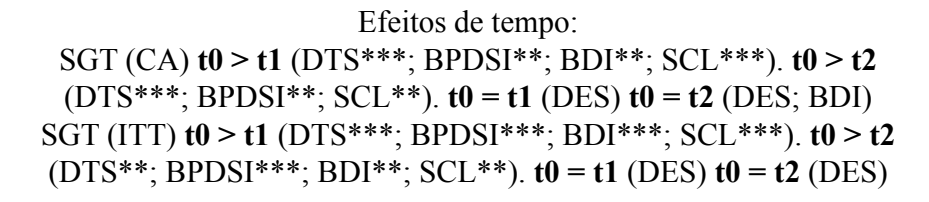 \\
\hline 07 & $\begin{array}{l}\text { EP } \\
\text { IS } \\
\text { PD }\end{array}$ & $\S \mathrm{TDI}=45$ & $\S 100$ & $\S 96,2$ & 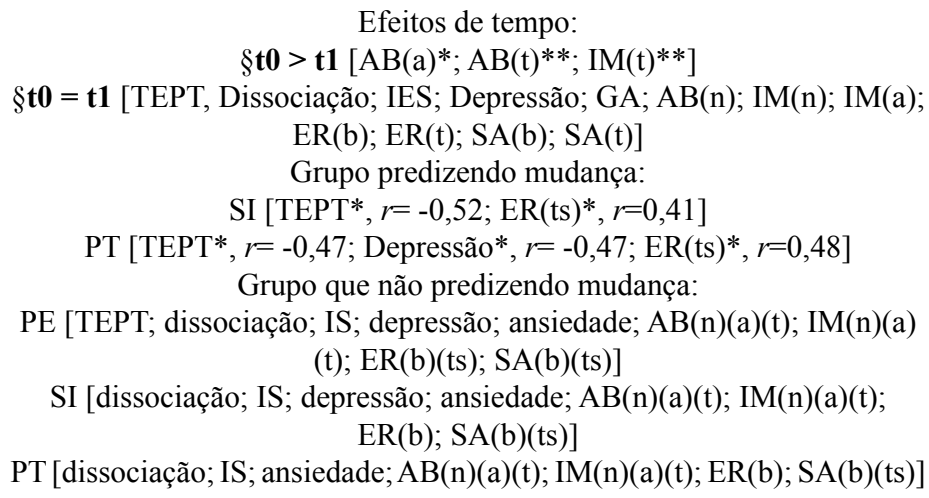 \\
\hline
\end{tabular}

Notas. FS $=$ Fobia Social; TD $=$ Transtorno Distímico; TDI $=$ Transtorno Dissociativo de Identidade TDM $=$ Transtorno Depressivo Maior; TP $=$ Transtorno do Pânico; TPB $=$ Transtorno de Personalidade Borderline; TPE $=$ Transtorno de Personalidade Esquiva; TUS = Transtorno por Uso de Substância. AF = Abuso Físico; AS = Abuso Sexual. ASI(a) = Addiction Severity Index - alcohol subsection; ASI(d) = Addiction Severity Index - drugs subsection; ASI(s) = Addiction Severity Index - social subsection; $\mathrm{AB}(\mathrm{a})=$ Attentional Bias - anxiety stimulus; $\mathrm{AB}(\mathrm{n})=$ Attentional Bias - neutral stimulus; $\mathrm{AB}(\mathrm{t})=$ Attentional Bias - trauma stimulus; BDI = Beck Depression Inventory; BPDSI = Borderline Personality Disorder Severity; BPRS = Brief Psychiatric Rating Scale $;$ CAPS = Clinical Administered PTSD Scale $;$ DES = Dissociative Experiences Scale $;$ DTS = Davidson Trauma Scale; $\mathrm{EM}(\mathrm{b})=$ Emotional Regulation - baseline; $\mathrm{ER}(\mathrm{ts})=$ Emotional Regulation - trauma slide $; \mathrm{GA}=$ general anxiety; HDRS = Hamilton Depression Rating Scale; IES = Impact of Event Scale; IES(av) = Impact of Event Scale - avoidance subscale $;$ IES(it) = Impact of Event Scale - intrusion subscale; $\operatorname{IM(a)~=~Implicit~Memory~-~anxiety~stimulus;~IM(n)~=~Im-~}$ plicit Memory - neutral stimulus; $\mathrm{IM}(\mathrm{t})=$ Implicit Memory - trauma stimulus; $\mathrm{IS}=$ Interpersonal Sensitivity; $\mathrm{SCL}=$ Symptom Checklist $;$ SIDES = Structured Interview for Disorders of Extreme Stress; $\mathrm{SA}(\mathrm{b})=$ Sympathetic Activation - baseline; $\mathrm{SA}(\mathrm{ts})=$ Sympathetic Activation - trauma slide; TSI = Trauma Symptom Inventory; TSI-DBS = Trauma Symptom Inventory- Dysfunctional Sexual Behavior Scale. $\mathrm{t} 0=$ pré-tratamento; $\mathrm{t} 1=$ pós-tratamento; $\mathrm{t} 2=$ follow-up $1 . \S=$ independente do tratamento; $\mathrm{nr}=$ não reportado; $\mathrm{AC}=$ Análise Completa; IT = Intenção de tratamento. Em destaque, diferenças entre os grupos experimentais e controle.

${ }^{*} p<0,05 ; * * p<0,01 ; * * * p<0,001$. 
Em geral, as intervenções terapêuticas adotadas pelos estudos revisados, independente do pressuposto teórico em que estão fundamentadas, foram capazes de reduzir os sintomas de $\mathrm{TC}$ ao longo do tempo. Embora haja benefícios entre os indivíduos que passam por intervenções usuais, recebem suporte psicológico ou permanecem em condições de espera por tratamento com o passar do tempo, quando são comparados a indivíduos que receberam tratamento através da abordagem da TCC, observa-se uma diferença significativa no que se refere a redução dos sintomas centrais de TEPT e TC. A TCC se mostrou mais efetiva para o tratamento de $\mathrm{TC}$, uma vez que foram encontradas diferenças significativas em prol da TCC nos estudos que realizaram análises de comparações entre os grupos no pós-tratamento (Cohen \& Hien, 2006; Dorrepaal et al., 2012; Trappler \& Newville, 2007; Zlotnick et al., 1997). Além disso, o estudo de Resick et al. (2003), único que fez uma comparação entre dois protocolos distintos de TCC, baseados na Exposição Prolongada e na Terapia Cognitiva Processual, não apontou diferenças significativas no que diz respeito a redução dos sintomas pós-traumáticos e depressivos.

A maioria dos protocolos utilizados pelos estudos revisados e que demonstraram resultados satisfatórios concentraram seu enfoque de tratamento nos sintomas afetivos e interpessoais (Dorrepaal et al., 2010; Dorrepaal et al., 2012; Trappler \& Newville, 2007; Zlotnick et al., 1997), o que sugere uma diferenciação destes protocolos com aqueles utilizados para o tratamento de TEPT, uma vez que possuem como objetivo intervir e auxiliar no desenvolvimento de habilidades de regulação emocional. As intervenções clássicas para TEPT são reconhecidas pelo enfoque na exposição ao trauma e, consequentemente, esbatimento dos sintomas associados por meio de dessensibilização e manejo da ansiedade (Butler et al., 2006). Além disso, observa-se um enfoque em abordar os sintomas dissociativos, somáticos e as possíveis alterações no sistema de crenças e significados do sujeito. Por exemplo, Dorrepaal et al. (2010; 2012), Trappler e Newville (2007) e Zlotnick et al. (1997), além dos sintomas pós-traumáticos e de regulação emocional, utilizaram técnicas de relaxamento e técnicas cognitivas para para identificação e modificação do sistema de crenças pós-traumáticas (ver Tabela 4 para resumo das principais técnicas cognitivo-comportamentais reportadas pelos estudos revisados).

Embora haja algumas particularidades entre os protocolos utilizados por estes quatro estudos, como por exemplo apenas o programa STAIR possuir sessões de exposição à história narrada do trauma, tanto o programa STAIR (Trappler $\&$ Newville, 2007) como as intervenções baseadas no manejo do afeto (Affect Management, em inglês) (Dorrepaal et al., 2010; 2012; Zlotnick et al., 1997) apresentaram resultados satisfatórios para o tratamento dos sintomas de TC. Estes resultados mostram-se em consonância com os achados prévios que revelaram uma melhora significativa nos sintomas pós-traumáticos de pacientes com TEPT crônico e histórico de abuso na infância a partir de intervenções focadas na regulação emocional (Cloitre et al., 2004; Cloitre et al., 2010; Mauritz, van de Sande, Goossens, van Achterberg, \& Draijer, 2014). Curiosamente, todos estes protocolos adaptados fundamentaram suas intervenções na modalidade de psicoterapia em grupos, não tendo sido verificados por esta revisão protocolos de TCC adaptados para o tratamento de TC na modalidade de psicoterapia individual.

Os estudos que adotaram a modalidade individual tiveram seus enfoques voltados para os sintomas centrais do TEPT e sintomas depressivos. Foram utilizadas abordagens com enfoque predominantemente comportamentais (Exposição Prolongada, Inoculação de Estresse) (Resick et al., 2003) e/ou cognitivas (Terapia Cognitiva Processual e Terapia Cognitiva Clássica) (Cohen \& Hien, 2006; Resick et al., 2003). Embora não se possa afirmar qual modalidade de intervenção psicoterapêutica conduz a desfechos mais favoráveis no tratamento de $\mathrm{TC}$, têm-se sugerido que abordar aspectos afetivos e interpessoais são essenciais para o tratamento destes indivíduos (Bryant, 2012; Cloitre et al., 2011; Cloitre et al., 2004; Herman, 2012; Mauritz et al., 2014). 


\section{Tabela 4}

Principais técnicas baseadas na terapia cognitivo-comportamental reportada pelos estudos

\begin{tabular}{|c|c|c|c|c|c|c|c|}
\hline & $\begin{array}{l}\text { Cohen } \\
\& \text { Hien } \\
(2006)\end{array}$ & $\begin{array}{l}\text { Dorrepaal } \\
\text { et al. } \\
(2012)\end{array}$ & $\begin{array}{l}\text { Dorrepaal } \\
\text { et al. } \\
(2010)\end{array}$ & $\begin{array}{l}\text { D’Andrea } \\
\text { \& Pole } \\
(2012)\end{array}$ & $\begin{array}{c}\text { Resick } \\
\text { et al. } \\
(2003)\end{array}$ & $\begin{array}{c}\text { Trappler } \\
\text { \& Newville } \\
(2007)\end{array}$ & $\begin{array}{c}\text { Zlotnick } \\
\text { et al. } \\
\text { (1997) }\end{array}$ \\
\hline Auto-regulação & $\mathrm{nr}$ & $\checkmark$ & $\checkmark$ & & & $\checkmark$ & $\checkmark$ \\
\hline Exposição in vivo & $\mathrm{nr}$ & & & & $\checkmark$ & & \\
\hline Exposição Imagistica & $\mathrm{nr}$ & & & $\checkmark$ & $\checkmark$ & & \\
\hline Exposição relato do trauma & $\mathrm{nr}$ & & & $\checkmark$ & $\checkmark$ & & \\
\hline Psicoeducação & $\mathrm{nr}$ & $\checkmark$ & $\checkmark$ & $\checkmark$ & & $\checkmark$ & $\checkmark$ \\
\hline Reestruturação Cognitiva & $\mathrm{nr}$ & $\checkmark$ & $\checkmark$ & $\checkmark$ & & $\checkmark$ & \\
\hline Repaternalização & $\mathrm{nr}$ & & & & & $\checkmark$ & \\
\hline Sono "seguro" & $\mathrm{nr}$ & $\checkmark$ & $\checkmark$ & & & & $\checkmark$ \\
\hline Tarefas de casa & $\mathrm{nr}$ & $\checkmark$ & $\checkmark$ & $\checkmark$ & $\checkmark$ & & $\checkmark$ \\
\hline Técnicas de distração & $\mathrm{nr}$ & & & & & $\checkmark$ & $\checkmark$ \\
\hline Técnicas de relaxamento & $\mathrm{nr}$ & $\checkmark$ & $\checkmark$ & $\checkmark$ & & $\checkmark$ & $\checkmark$ \\
\hline Treino de Assertividade & $\mathrm{nr}$ & $\checkmark$ & $\checkmark$ & & & $\checkmark$ & \\
\hline
\end{tabular}

Nota. nr = não reportado.

Algumas questões metodológicas devem ser consideradas para análise e conclusão dos resultados levantados através desta revisão. O número limitado de estudos com amostras com TC ou com sintomas de TC é um fator que prejudica a extensão de nossas conclusões. No entanto, apesar da revisão ter elegido um número reduzido de estudos, as buscas foram conduzidas em três bases de dados internacionalmente consolidadas. Além disso, não houve restrições quanto ao período de tempo e foram utilizados prioritariamente termos MeSH (Medical Subject Headings). Embora a revisão tenha considerado apenas artigos em inglês, fato que pode influenciar o número de artigos incluídos, o diagnóstico de TC ainda é muito pouco discutido no Brasil, tendo sido publicados recentemente apenas artigos de revisão e metodológicos em lingua portuguesa (Camargo, Kluwe-Schiavon, Sanvicente-Vieira, Levandowski, \& GrassiOliveira, 2013; Viola et al., 2011).

Outra limitação refere-se a heterogeneidade entre as amostras dos estudos incluídos. Mesmo que os participantes tenham diagnósti- co de TEPT e tenham sido vítimas de múltiplos traumas ao longo da vida, a presença de outros diagnóstico na amostra pode ser um viés importante ao se comparar e interpretar os efeitos de diferentes intervenções. O estudo de Trappler e Newville (2007), por exemplo, foi realizado com uma população com diagnóstico de Esquizofrênia e Transtorno de Personalidade Esquizoafetivo. Sabe-se que portadores destes diagnósticos possuem respostas distintas a intervenções terapêuticas em comparação a outros diagnósticos, uma vez que possuem limitações cognitivas decorrentes da cronicidade do transtornos (Kluwe-Schiavon, Sanvicente-Vieira, Kristensen, \& Grassi-Oliveira, 2013). Além dos diagnósticos conhecidos presentes nas amostras, os estudos revisados demonstram altas taxas de comorbidades entre os participantes. Este dado pode influenciar a resposta dos participantes as intervenções e, por consequência, enviesar os efeitos do tratamento. As principais comorbidades encontradas (i.e., TDM e TPB) referem-se aquelas sugeridas como possíveis diagnósticos comórbidos ao TEPT, mas que de fato poderiam ser com- 
preendidas como parte de um quadro complexo em resposta a exposição a estressores repetidos durante fases iniciais do desenvolvimento.

É consenso que experiências adversas durante períodos precoces do desenvolvimento podem levar a alterações neurobiológicas, cognitivas e comportamentais irreversíveis e causar impacto no funcionamento do indivíduo na vida adulta (Andersen \& Teicher, 2009; Teicher, Andersen, Polcari, Anderson, \& Navalta, 2002; Teicher et al., 2003). Portanto, por se tratar de um transtorno que se desenvolve a partir da exposição recorrente a estressores durante o desenvolvimento, é esperado que na ausência de um diagnóstico acurado de $\mathrm{TC}$, o indivíduo receba múltiplos diagnósticos. Ainda, referente as características das amostras dos estudo incluídos na revisão, o fato dos artigos serem predominantemente compostos apenas por amostras femininas é outra limitação para a interpretação dos resultados, pois não se sabe como seria a resposta de tais intervenções em amostras masculinas.

A falta de descrição dos protocolos de intervenção adotados também foi um problema encontrado entre os estudos incluídos. Somente os estudos de Dorrepaal et al. $(2010$; 2012) e Trappler e Newville (2007) descreveram os tópicos principais a serem abordados entre as sessões ou entre as fases do tratamento. Não obstante, observou-se uma variabilidade no que se refere a seleção dos instrumentos de avaliação para sintomas de TC. Alguns estudos utilizaram de escalas de sintomas psiquiátricos gerais (Dorrepaal et al., 2010; Trappler \& Newville, 2007), enquanto outras selecionaram escalas para avaliar sintomas especificos e centrais ao diagnóstico. Curiosamente, a SIDES, instrumento próprio para diagnóstico e avaliação de sintomas de TC, foi utilizada apenas por Dorrepaal et al. (2010; 2012). A carência de instrumentos de avaliação evidência a escassez de estudos com TC, sendo necessário o desenvolvimento de escalas e inventários que possibilitem uma avaliação mais acurada de seus sintomas e, consequentemente, uma melhor interpretação dos resultados decorrentes de intervenções. Outros sintomas que compreendem a proposta diagnóstica de $\mathrm{TC}$, como alterações no funcionamento cognitivo e sintomas somáticos, foram negligênciados entre a maioria dos estudos. A execessão foi o trabalho de D'Andrea e Pole (2012) que utilizou tarefas para avaliar a regulação emocional, viés atencional e memória implícita.

Levando em consideração os artigos analisados nesta revisão, pode-se concluir que ainda são incipientes os estudos empíricos no que se refere ao tratamento de indivíduos com diagnóstico de TC, bem como os instrumentos que permitem a avaliação diagnóstica e/ou dos sintomas subjacentes ao transtorno. Constatou-se ainda que o diagnóstico de TC têm sido mais considerado no campo teórico do que na prática clínica, o que também influencia na sua baixa aplicabilidade clínica. Além disso, acredita-se que muitos dos estudos já publicados com amostras compostas por sujeitos diagnosticados com TEPT e com história de trauma na infância podem, na verdade, se referir a diagnóstico de de TC e, desta forma, corroborar para a escassez dos achados sobre intervenções para este transtorno.

Em relação as intervenções baseadas na TCC revisadas, cabe destacar que somente em dois casos foram usadas técnicas de exposição. Tais técnicas são consolidadas para o tratamento de pacientes com TEPT (Foa, Hembree, \& Rothbaum, 2007), demonstrando resultados satisfatórios no esbatimento dos sintomas póstraumáticos. No entanto ao que parece para pacientes com TC têm-se optado pelo uso de técnicas direcionadas à auto-regulação, reestruturação cognitiva e manejo de ansiedade. Apesar disso, para que maiores conclusões possam ser feitas no que tange ao enfoque cognitivo-comportamental mais adequado para o tratamento de TC ainda necessita-se de avanços no conhecimento sobre o intervenções para este transtorno. Assim, estudos clínicos controlados e randomizados podem ser alternativas para investigar a eficácia de diferentes abordagens dentro das TCCs, como por exemplo, intervenções com técnicas de exposição $\mathrm{X}$ intervenções direcionadas à reestruturação cogntiva e auto-regulação. Futuros estudos também devem buscar uma melhor definição de TC para seleção de suas amostras, utilizando-se 
prioritariamente de instrumentos de avaliação de TC e não só fundamentando-se em instrumentos usados para avaliação de TEPT.

Em síntese, os achados da presente revisão convergem para benefícios de intervenções, independente do pressuposto teórico, no tratamento de sintomas de TC. Ainda, foi possível identificar em alguns dos estudos elegíveis que a TCC apresenta melhores resultados em comparação aos tratamentos usuais/controle. Além disso, tanto a TCC em grupo como individual demonstram-se recomendadas para o tratamento de sintomas de TC.

\section{Referências}

American Psychiatric Association. (1994). Diagnostic and statistical manual of mental disorders ( $4^{\text {th }}$ ed.). Washington, DC: Author.

American Psychiatric Association. (2013). Diagnostic and statistical manual of mental disorders ( $5^{\text {th }}$ ed.). Washington, DC: Author.

Andersen, S. L., \& Teicher, M. H. (2009). Desperately driven and no brakes: Developmental stress exposure and subsequent risk for substance abuse. Neuroscience \& Biobehavioral Reviews, 33(4), 516-524. doi:10.1016/j.neubiorev.2008.09.009

Bryant, R. A. (2012). Simplifying complex PTSD: Comment on Resick et al. (2012). Journal of Traumatic Stress, 25(3), 252-253, discussion on 260-253. doi:10.1002/jts.21696

Butler, A. C., Chapman, J. E., Forman, E. M., \& Beck, A. T. (2006). The empirical status of cognitive-behavioral therapy: A review of meta-analyses. Clinical Psychology Review, 26(1), 17-31. doi:10.1016/j.cpr.2005.07.003

Camargo, J., Kluwe-Schiavon, B., Sanvicente-Vieira, B., Levandowski, M. L., \& Grassi-Oliveira, R. (2013). Brazilian version of the Structured Interview for Disorders of Extreme Stress - Revised (SIDES-R): Adaptation and validation process. Trends in Psychiatry and Psychotherapy, 35(4), 292-298.

Cloitre, M., Cohen, L. R., \& Koenen, K. C. (2006). Treating survivors of childhood abuse. London: Guilford Press.

Cloitre, M., Courtois, C. A., Charuvastra, A., Carapezza, R., Stolbach, B. C., \& Green, B. L. (2011). Treatment of complex PTSD: Results of the ISTSS expert clinician survey on best practices. Journal of Traumatic Stress, 24(6), 615-627. doi:10.1002/jts.20697
Cloitre, M., Stolbach, B. C., Herman, J. L., van der Kolk, B., Pynoos, R., Wang, J., \& Petkova, E. (2009). A developmental approach to complex PTSD: Childhood and adult cumulative trauma as predictors of symptom complexity. Journal of Traumatic Stress, 22(5), 399-408. doi:10.1002/ jts. 20444

Cloitre, M., Stovall-McClough, K. C., Miranda, R., $\&$ Chemtob, C. M. (2004). Therapeutic alliance, negative mood regulation, and treatment outcome in child abuse-related posttraumatic stress disorder. Journal of Consulting and Clinical Psychology, 72(3), 411-416. doi:10.1037/0022-006X.72.3.411

Cloitre, M., Stovall-McClough, K. C., Nooner, K., Zorbas, P., Cherry, S., Jackson, C. L., ...Petkova, E. (2010). Treatment for PTSD related to childhood abuse: A randomized controlled trial. American Journal of Psychiatry, 167(8), 915924. doi:10.1176/appi.ajp.2010.09081247

Cohen, L. R., \& Hien, D. A. (2006). Treatment outcomes for women with substance abuse and PTSD who have experienced complex trauma. Psychiatric Services, 57(1), 100-106. doi:10.1176/appi. ps.57.1.100

Courtois, C. A., Ford, J. D., \& Cloitre, M. (2009). Treating complex traumatic stress disorders: An evidence based guide. In C. A. Courtois \& J. D. Ford (Eds.), Best practices in psychotherapy for adults (pp. 82-103). New York: The Guilford Press.

D’Andrea, W.,\&Pole,N.(2012). Anaturalistic study of the relation of psychotherapy process to changes in symptoms, information processing, and physiological activity in Complex Trauma. Psychological Trauma: Theory, Research, Practice, and Policy, 4(4), 438-446. doi:10.1037/a0025067

Dorrepaal, E., Thomaes, K., Smit, J. H., van Balkom, A. J., van Dyck, R., Veltman, D. J., \& Draijer, N. (2010). Stabilizing group treatment for Complex Posttraumatic Stress Disorder related to childhood abuse based on psycho-education and cognitive behavioral therapy: A pilot study. Child Abuse \& Neglect, 34(4), 284-288. doi:10.1016/j.chiabu.2009.07.003

Dorrepaal, E., Thomaes, K., Smit, J. H., van Balkom, A. J., Veltman, D. J., Hoogendoorn, A. W., \& Draijer, N. (2012). Stabilizing group treatment for complex posttraumatic stress disorder related to child abuse based on psychoeducation and cognitive behavioural therapy: A multisite randomized controlled trial. Psychotherapy and Psychosomatics, 81(4), 217-225. doi: $10.1159 / 000335044$ 
Foa, E. B., Hembree, E. A., \& Rothbaum, B. O. (2007). Prolonged exposure therapy for PTSD: Emotional processing of traumatic experiences, therapist guide. New York: Oxford University Press.

Herman, J. L. (1992). Complex PTSD: A syndrome in survivors of prolonged and repeated trauma. Journal of Traumatic Stress, 5, 377-391. doi:10.1002/jts.2490050305

Herman, J. (2012). CPTSD is a distinct entity: Comment on Resick et al. (2012). Journal of Traumatic Stress, 25(3), 256-257, discussion on 260253. doi:10.1002/jts. 21697

Kluwe-Schiavon, B., Sanvicente-Vieira, B., Kristensen, C. H., \& Grassi-Oliveira, R. (2013). Executive functions rehabilitation for schizophrenia: A critical systematic review. Journal of Psychiatric Research, 47(1), 91-104. doi:10.1016/j.jpsychires.2012.10.001

Mauritz, M. W., van de Sande, R., Goossens, P. J., van Achterberg, T., \& Draijer, N. (2014). Phase-based treatment of a complex severely mentally ill case involving complex posttraumatic stress disorder and psychosis related to Dandy Walker syndrome. Journal of Trauma \& Dissociation, 15(5), 588-606. doi:10.1080/15299732.2014.938213

McLean, L. M., \& Gallop, R. (2003). Implications of childhood sexual abuse for adult borderline personality disorder and complex posttraumatic stress disorder. American Journal of Psychiatry, 160(2), 369-371. doi:10.1176/appi. ajp.160.2.369

Pelcovitz, D., van der Kolk, B., Roth, S., Mandel, F., Kaplan, S., \& Resick, P. (1997). Development of a criteria set and a structured interview for disorders of extreme stress (SIDES). Journal of Traumatic Stress, 10(1), 3-16.

Resick, P. A., Bovin, M. J., Calloway, A. L., Dick, A. M., King, M. W., Mitchell, K. S., ...Wolf, E. J. (2012). A critical evaluation of the complex PTSD literature: Implications for DSM-5. Journal of Traumatic Stress, 25(3), 241-251. doi:10.1002/jts.21699

Resick, P. A., Nishith, P., \& Griffin, M. G. (2003). How well does cognitive-behavioral therapy treat symptoms of complex PTSD? An examination of child sexual abuse survivors within a clinical trial. CNS Spectrums, 8(5), 340-355.

Roth, S., Newman, E., Pelcovitz, D., van der Kolk, B., \& Mandel, F. S. (1997). Complex PTSD in victims exposed to sexual and physical abuse: Results from the DSM-IV Field Trial for Posttraumatic Stress Disorder. Journal of Traumatic Stress, 10(4), 539-555.
Teicher, M. H., Andersen, S. L., Polcari, A., Anderson, C. M., \& Navalta, C. P. (2002). Developmental neurobiology of childhood stress and trauma. Psychiatric Clinics of North America, 25(2), 397-426, vii-viii.

Teicher, M. H., Andersen, S. L., Polcari, A., Anderson, C. M., Navalta, C. P., \& Kim, D. M. (2003). The neurobiological consequences of early stress and childhood maltreatment. Neuroscience \& Biobehavioral Reviews, 27(1-2), 33-44. doi:10.1016/S0149-7634(03)00007-1

Teicher, M. H., \& Samsom, J. A. (2013). Childhood maltreatment and psychopathology: A case for ecophenotypic variants as clinically and neurobiologically distinct subtypes. American Journal of Psychiatry, 170(10), 1114-1133. doi:10.1176/ appi.ajp.2013.12070957

Trappler, B., \& Newville, H. (2007). Trauma healing via cognitive behavior therapy in chronically hospitalized patients. Psychiatric Quarterly, 78(4), 317-325. doi:10.1007/s11126-007-90498

Van der Kolk, B. A. (2005). Developmental trauma disorder: Toward a rational diagnosis for children with complex trauma histories. Psychiatric Annals, 35, 401-408.

Van der Kolk, B. A., Pelcovitz, D., Roth, S., Mandel, F. S., McFarlane, A., \& Herman, J. L. (1996). Dissociation, somatization, and affect dysregulation: The complexity of adaptation of trauma. American Journal of Psychiatry, 153(7 Suppl.), 83-93. doi:10.1176/ajp.153.7.83

Van der Kolk, B. A., Roth, S., Pelcovitz, D., Sunday, S., \& Spinazzola, J. (2005). Disorders of extreme stress: The empirical foundation of a complex adaptation to trauma. Journal of Traumatic Stress, 18(5), 389-399. doi:10.1002/jts.20047

Viola, T. W., Kluwe-Schiavon, B., Renner, A. M., \& Grassi-Oliveira, R. (2011). Trauma complexo e suas implicações diagnósticas. Revista de Psiquiatria do Rio Grande do Sul, 33(1), 55-62. doi:10.1590/S0101-81082011000100010

Zlotnick, C., Shea, T. M., Rosen, K., Simpson, E., Mulrenin, K., Begin, A., \& Pearlstein, T. (1997). An affect-management group for women with posttraumatic stress disorder and histories of childhood sexual abuse. Journal of Traumatic Stress, 10(3), 425-436. 